\title{
A computational study of the aerodynamic performance of a dragonfly forewing in gliding flight
}

\author{
Huixiang Liu', Guoyi He${ }^{2}$, Chengyu Ma ${ }^{1}$, Qi Wang ${ }^{3}$ and Yun Luo ${ }^{1}$ \\ ${ }^{1}$ Nanchang Hangkong University School of Aircraft Engineering,student,2017, China \\ ${ }^{2}$ Nanchang Hangkong University School of Aircraft Engineering, associate professor, 2017, China \\ ${ }^{3}$ Nanchang Hangkong University School of Aircraft Engineering, professor,2017, China
}

\begin{abstract}
Gliding flight is a common mode of flight for dragonfly, the objective of the current research is to use numerical simulations to explore whether the corrugations have positive effect on aerodynamic performance of the dragonfly wings in gliding flight. In order to compare aerodynamic performance of the dragonfly wing and flat plate, a three-dimensional model of the dragonfly forewing and a three-dimensional flat plate with the same shape of the dragonfly forewing are established. The flow fields around three-dimensional dragonfly forewing and flat plate are simulated for $\mathrm{Re}=10000$ and angles of attack changing from $0^{\circ}$ to $25^{\circ}$ (with an interval of $5^{\circ}$ ), numerical simulation indicate that aerodynamic performance of the dragonfly wing is slightly better than the flat plate over the entire range of parameters tested, especially the effect of the corrugateions on the flow is more evident at large angle of attack.
\end{abstract}

\section{Introduction}

Dragonflies, as one of the best flying insects in nature, are capable of carrying out dramatic flight manoeuvres. They can hover, accelerate in almost any direction and manoeuvre precisely at high speed to intercept other insects[1], however, gliding flight is also an important flight mode of the dragonflies[2], especially at high temperature, in order to avoid higher heat quantity in body[3], gliding flight is observed more frequently in dragonflies. It is interest that dragonflies could keep only a pair of forewings beat frequency and hindwings are immovable, or keep all of wings stationary in gliding flight.

The incredible flight ability produced by dragonflies is through the flapping of their forewings and hindwings. Unlike typical engineered airfoil, dragonfly wings are not smooth, wing cross-section are highly corrugated. Plated wings vary across the wing span and chord, it has been shown that corrugations could reduce the weight of the wings[4], improving the stability of the wings[5] and enhance the spanwise stiffness in the wings[6]. Howerer, whether the corrugations can improve the aerodynamic performance of the dragonfly wings in gliding flight,there is a difference in the current study.

Experimental measurements of the aerodynamic effect of corrugations were carried out by Kesel[7], Kesel have shown that corrugations plays an important role in the lift production of dragonfly wings,similar results were also found from a computational study corresponding to the same configuration has undertaken by Vargas[2]. On the contrary,early wind tunnel experiments of inset wings conducted by Reese[8]concluded that the corrugations has no aerodynamic significance, a computational study of the aerodynamic effect of corrugations were taken by Sun [9] both concluded that the effect of corrugations were to decrease the lift, the lift of corrugated wings are less than the lift of flat plate. There is still no consensus on whether corrugated wings perform better than flat plate, their research was limited comparing between the aerodynamic performance of different dragonfly wing cross-sections and two-dimensional flat plate. In fact, experimental measurements[7][10]of dragonfly wing cross-sections shown that corrugations vary with the relative span length.

It is this reason that makes further investigation into the aerodynamic aspect of dragonflies' wing worthwhile, in order to provide some perspective for the different performance between the corrugated wings and flat plate, this study focus on whether the corrugations have positive effect on aerodynamic performance of the dragonfly wings in gliding flight.

\section{Numerical method}

\subsection{Geometric models of dragonfly wing}

Dragonfly wings are complex in structure,composed of thin cuticular membrane that is supported by a system of veins, moreover, the thickness of thin cuticular membrane are only 4 microns [11], veins can be divided into longitudinal veins and crossveins, It is possible to construct the three-dimensional model of the dragonfly wings according to the actual measured data by 
Okamoto[10]and Ren[12]. In order to simplify the model, influence of the irregular shape of the veins, impact of the extremely tiny corrugations, size variation of the wing along the span and chord and other structures like pterostigma, nodus etc. were ignored.The geometry of the dragonfly forewing was established by using the 3D modeling software through reverse engineering. The size of the veins are $0.035 \mathrm{~mm}$, veins are shown in Figure 1, The veins are filled with $0.004 \mathrm{~mm}$ cuticular membrane as in Figure 2, the shape of cuticular membrane is from the forewing of dragonfly in nature. Figure 3 shows the three-dimensional model of the dragonfly forewing, the spatial distribution of veins and cuticular membrane forms corrugation.

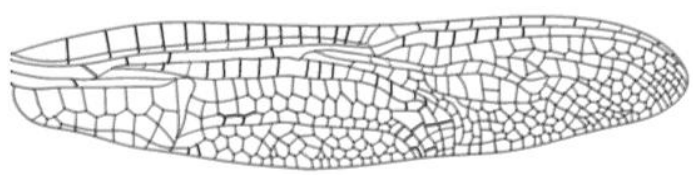

Figure 1. Veins of dragonfly forewing

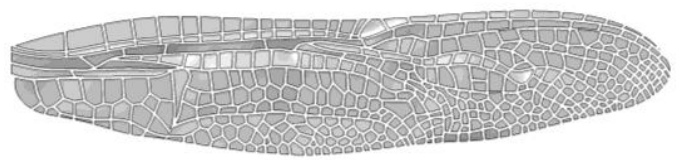

Figure 2. Cuticular membrane

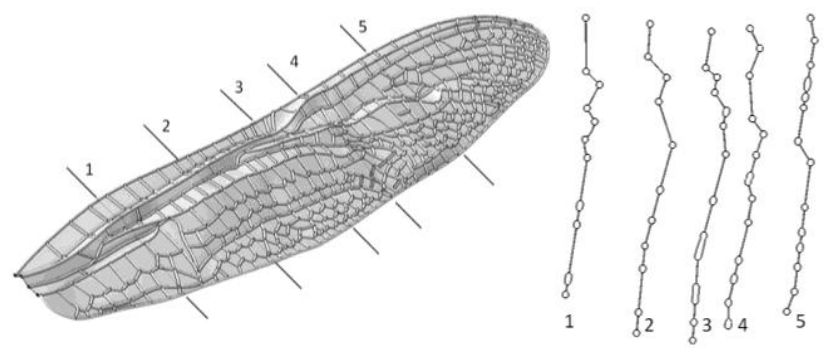

Figure 3. Three-dimensional model of the dragonfly forewing

In order to answer such a question: whether the corrugations have positive effect on aerodynamic performance of the dragonfly wings in gliding flight, a three-dimensional flat plate with the same shape and reference values as the dragonfly forewing was built, and the thickness of the flat plate is $0.035 \mathrm{~mm}$, as shown in Figure 4.

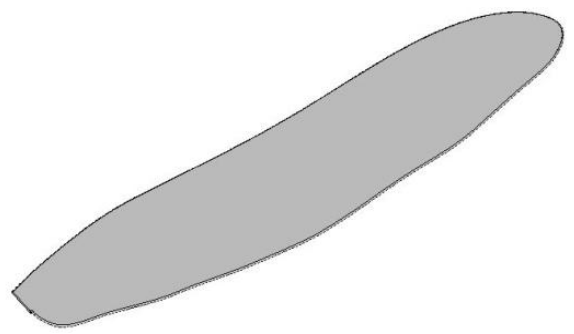

Figure 4. Three-dimensional flat plate

\subsection{Governing equations}

The incompressible 3D Navier-Stokes equations are follows:

$$
\begin{aligned}
& \frac{\partial u}{\partial v}+\frac{\partial v}{\partial y}+\frac{\partial w}{\partial y}=0 \\
& \frac{\partial u}{\partial t}+u \frac{\partial u}{\partial x}+v \frac{\partial u}{\partial y}+w \frac{\partial u}{\partial y}=-\frac{\partial p}{\partial x}+\frac{1}{\operatorname{Re}}\left(\frac{\partial^{2} u}{\partial x^{2}}+\frac{\partial^{2} u}{\partial y^{2}}+\frac{\partial^{2} u}{\partial z^{2}}\right) \\
& \frac{\partial v}{\partial t}+u \frac{\partial v}{\partial x}+v \frac{\partial v}{\partial y}+w \frac{\partial v}{\partial y}=-\frac{\partial p}{\partial y}+\frac{1}{\operatorname{Re}}\left(\frac{\partial^{2} v}{\partial x^{2}}+\frac{\partial^{2} v}{\partial y^{2}}+\frac{\partial^{2} v}{\partial z^{2}}\right) \\
& \frac{\partial w}{\partial t}+u \frac{\partial w}{\partial x}+v \frac{\partial w}{\partial y}+w \frac{\partial w}{\partial y}=-\frac{\partial p}{\partial y}+\frac{1}{\operatorname{Re}}\left(\frac{\partial^{2} w}{\partial x^{2}}+\frac{\partial^{2} w}{\partial y^{2}}+\frac{\partial^{2} w}{\partial z^{2}}\right)
\end{aligned}
$$

Where $u, v$ and $w$ are the non-dimensionalized components of velocity along $x, y$ and $z$ directions; $P$ and $t$ is the non-dimensional fluid pressure and time, The equations are non-dimensionalized. corresponds to the Reynolds number defined as:

$$
\operatorname{Re}=\frac{\rho U c}{\mu}=\frac{U c}{v}
$$

Where $\rho, \mathrm{U}$ and $c$ denote respectively the density, the free stream velocity and length, and represent the dynamic viscosity coefficient and the kinematic viscosity coefficient respectively.

The key examined are the lift and drag coefficient which are defined as

$$
\mathrm{Cl}=\mathrm{F}_{1} / 0.5 \rho U^{2} c \quad \mathrm{Cd}=\mathrm{F}_{\mathrm{d}} / 0.5 \rho U^{2} c
$$

Where $F_{1}$ and $F_{d}$ are the lift and drag of an airfoil respectively.

\subsection{Boundary conditions}

The three-dimensional domains implemented in the current study consist of six boundary conditions located front, back, left, right, top and bottom of the computational domain, the size of the computational domain is shown in Figure 5, the boundary conditions of left field and the dragonfly forewing are symmetry and wall respectively.

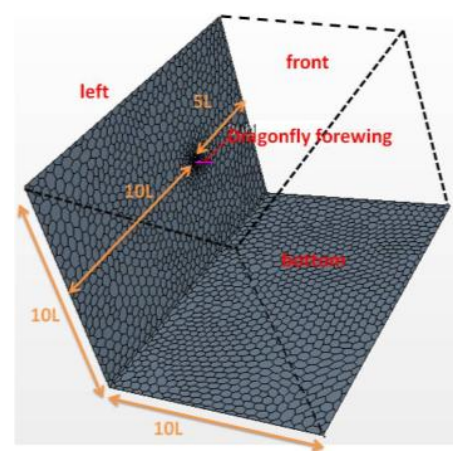

Figure 5. Computational domains 


\subsection{Validation case}

In order to validate that the current numerical solver do not depend on the selection of the grid, we build three type of grids : the first type of the grid is that minimum size of the grid was $0.04 \mathrm{~mm}$, the second type of the grid is that minimum size of the grid was $0.03 \mathrm{~mm}$, the third type of the grid is that minimum size of the grid was 0.02 $\mathrm{mm}$. The results show that the relative error of the lift coefficient is $14.9 \%$ in the first and the second type of grid, and the relative error of the second and the third type of gird is $4.7 \%$. The difference in the lift coefficient is no more than $5 \%$, that is an acceptable value. Considering a balance between accuracy and efficiency of the numerical simulation, we chose second type of grid as the solution of grid, as shown in Figure 6.
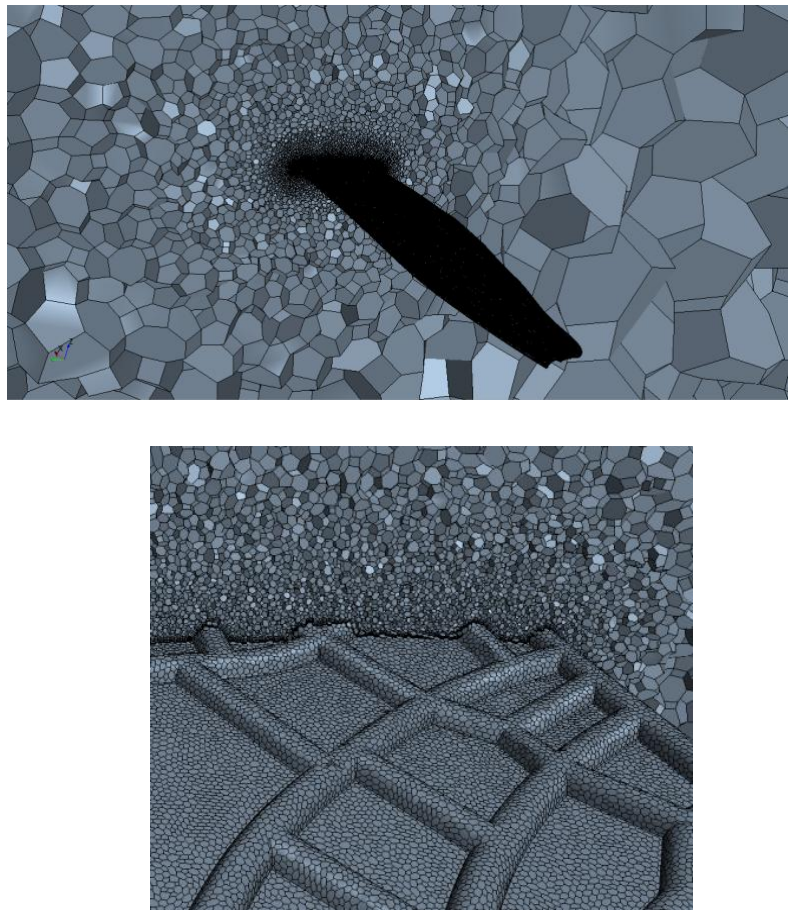

Figure 6. Local showing of computational grid.

Six validation cases were conducted using threedimensional flat plate at angle of attack of $0^{\circ}$ to $25^{\circ}$ (with an interval of $5^{\circ}$ )with $\mathrm{Re}=10000$, The simulations were performed on second gird for $0^{\circ}$ to $25^{\circ}$ angle of attack cases respectively.The comparison between the experimental and force coefficient in Figure 7 show indistinctive discrepancies between the two results. The numerical lift coefficient is close to the experimental value at angle of attack of $0^{\circ}$ to $10^{\circ}$, the numerical lift coefficient is slightly larger than the experiment value at angle of attack of $15^{\circ}$ and $20^{\circ}$, The numerical drag coefficient is close to the experimental value at angle of attack of $0^{\circ}$ to $25^{\circ}$, the numerical drag coefficient is slightly smaller than the experiment value at the other angle of attack. It should be noticed that the overall trend of two results is consistent and the maximum relative error is below $10 \%$.

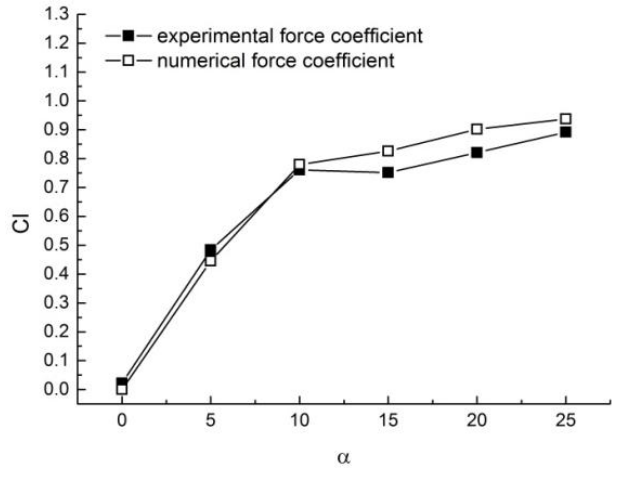

(a)

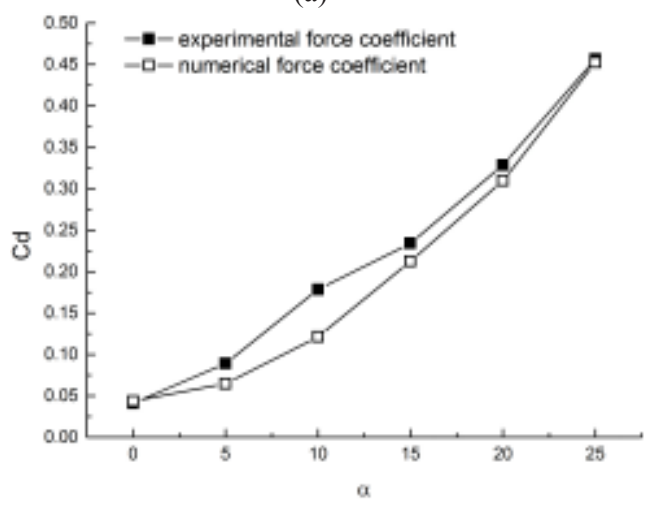

(b)

Figure 7. Comparison between the numerical and the experimental force coefficient of Kesel for $\mathrm{Re}=10000$ and various anglesof attack.(a)lift coefficient,(b)drag coefficient.

\section{Results}

The flow fields around three-dimensional dragonfly forewing and flat plate are simulated for $\mathrm{Re}=10000$ and angles of attack changing from $0^{\circ}$ to $25^{\circ}$ (with an interval of $5^{\circ}$ ), the following sections discuss the force coefficient of three-dimensional dragonfly forewing and flat plate obtained from numerical simulation.The lift and drag coefficient curves for dragonfly forewing and flat plate are shown in Figure 8, it was found from Figure 8 that lift coefficient of dragonfly forewing is slightly higher than the flat plate at all angle of attack, however, drag coefficient of dragonfly forewing is slightly higher than the flat plate at all angle of attack.

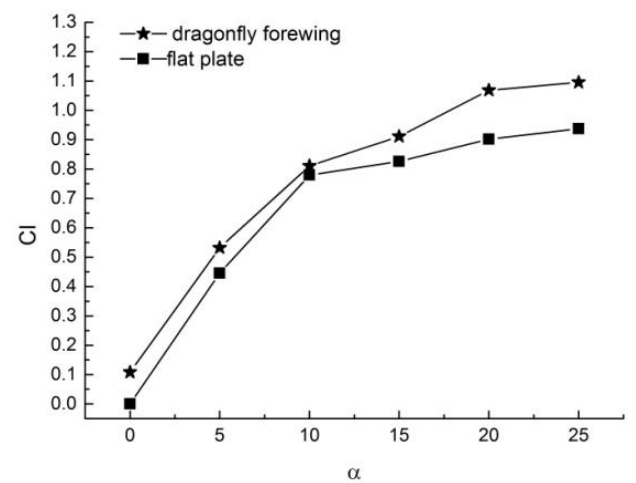

(a) 


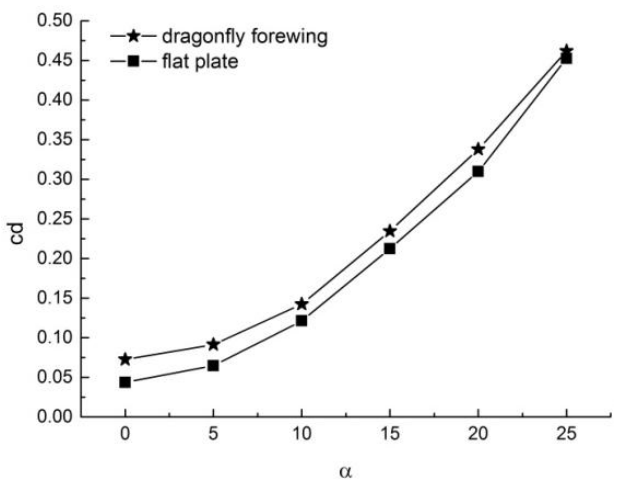

(b)

Figure 8. Aerodynamic performance of dragonfly forewing and flat plate (a)lift coefficient, (b)drag coefficient.

Figure 9(a) and (b) show that there is a trapped vortex in each fold which causes the overall flow to resemble the smooth airfoil. The higher lift coefficient of dragonfly forewing due to these trapped vortexes inside each corrugation, it is important these corrugations delayed flow separation so that the lift coefficient of dragonfly forewing are more higher than flat plate at large angle of attack, however, the higher drag coefficient of dragonfly forewing are produced because of these corrugations.

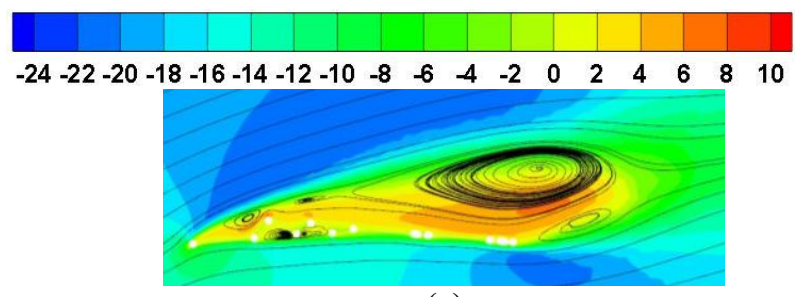

(a)

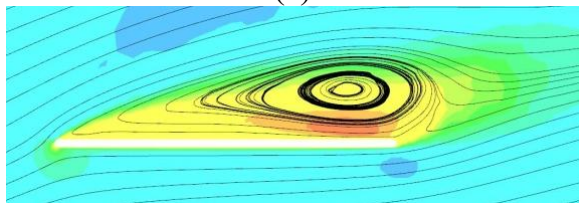

(b)

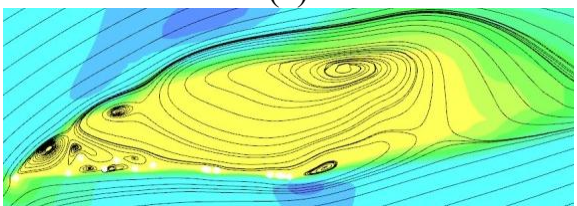

(c)

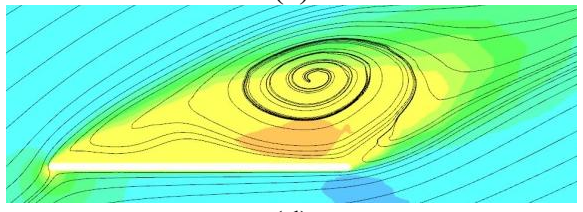

(d)

Figure 9. The streamline of airfoil with profile cross sections shown below at $0.21 \mathrm{lel}$, where lrel is the relative span length: (a) and (c) are dragonfly forewing at angles of attack $15^{\circ}$ and $20^{\circ}$ respectively; (b) and (d) are flat plate at angles of attack $15^{\circ}$ and $20^{\circ}$ respectively.

A leading edge vortex(LEV) is formed on first longitudinal vein of dragonfly forewing as shown in Figure10(a), A LEV is also formed on the tips of flat plate as shown in Figure10(b), Figure 10 show that the
LEVs of dragonfly forewing is much higher than the LEVs of flat plate at same condition, so LEVs should be a source of high lift forces in dragonflies.

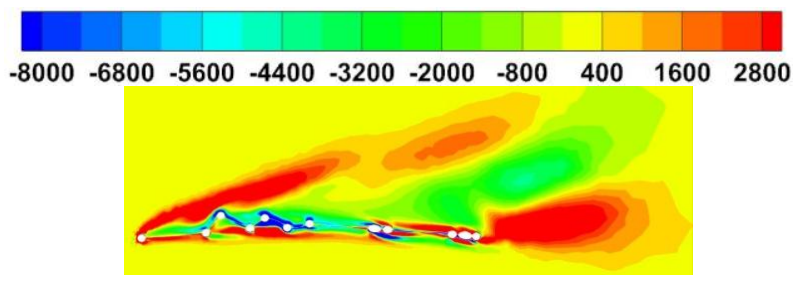

(a)

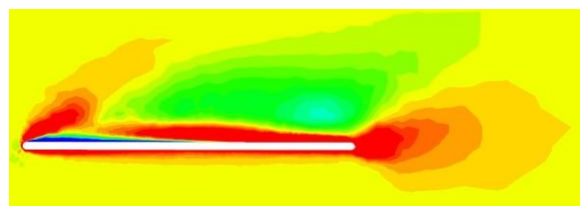

(b)

Figure 10. Vortex of airfoil angles of attack $15^{\circ}$ with profile cross sections shown below at $0.21 \mathrm{rel}$, where lrel is the relative span length: (a) dragonfly forewing (b) flat plate.

\section{Conclusion}

Dragonflies have developed perfect wings through millions of years of adaptation,despite highly corrugated dragonfly forewing, it is found that the aerodynamic performance of the dragonfly wing is slightly better than the flat plate over the entire range of parameters tested, especially the effect of the corrugateions on the flow is more evident at large angle of attack.

This result can be a potential inspiration to the airfoil design of micro-aerial vehicles(MAVs), in this respect, nature provides a perfect design of wings that are suitable for flapping MAVs.

\section{References}

1. A. L. Thomas, G. K. Taylor, R. B. Srygley, J Exp Biol. J. 207, 4299 (2004)

2. A. Vargas, R. Mittal, H. Dong, Bioinspir Biomim. J.3, 13-0 ( 2008)

3. R. Xiujuan, Scientia Sinica. J. 56,884-897 (2013)

4. S. Sudo, K. Tsuyuki, T. Ikohagi, JSME Int J.J. 42,721-729(1999)

5. H. Hertel, Structure, form, movement.M.(1966)

6. B. K. Antonia, P. Ute, N. Werner, Comput Biol Med. J. 28,423-437(1998)

7. A. B. Kesel, J Exp Biol. J.203,31253-3135(2000)

8. C. J. C.Rees, Nat.J. 200-203 (1975)

9. X. G. Meng, M. Sun, Phy Flu.J. 25, 200 ( 2013)

10. M. Okamoto, K. Yasuda,A. Azuma, J Exp Biol.J. 199,281-294(1996)

11. J. G. Needham,A genealogic study of dragonfly wing vention.M. 703-706 (1993)

12. H. H. Ren,X. S. Wang,Y. L. Chen, Chin Phys B.J. 21, 294-303 (2012) 